\title{
AgOnt: Ontology for Agriculture Internet of Things
}

\author{
Siquan $\mathrm{Hu}^{1}$, Haiou Wang ${ }^{1}$, Chundong $\mathrm{She}^{2}$, and Junfeng Wang ${ }^{3}$ \\ ${ }^{1}$ School of Information Engineering, University of Science and Technology Beijing, \\ Beijing 100083, P.R. China \\ ${ }^{2}$ University of Electronic Science and Technology of China \\ ${ }^{3}$ College of Computer Science, Sichuan University \\ husiquan@ustb.edu.cn
}

\begin{abstract}
Recent advances in networking and sensor technologies allow various physical world objects connected to form the Internet of Things (IOT). As more sensor networks are being deployed in agriculture today, there is a vision of integrating different agriculture IT system into the agriculture IOT. The key challenge of such integration is how to deal with semantic heterogeneity of multiple information resources. The paper proposes an ontology-based approach to describe and extract the semantics of agriculture objects and provides a mechanism for sharing and reusing agriculture knowledge to solve the semantic interoperation problem. AgOnt, ontology for the agriculture IOT, is built from agriculture terminologies and the lifecycles including seeds, grains, transportation, storage and consumption. According to this unified meta-model, heterogeneous agriculture data sources can be integrated and accessed seamlessly.
\end{abstract}

Keywords: Agriculture Internet of Things, Ontology, Semantics.

\section{Introduction}

Recent advances in networking, sensor and RFID technologies allow connecting various physical world objects to the IT infrastructure, which could, ultimately, enable realization of the Internet of Things (IOT) and the Ubiquitous Computing visions [1] [2] [3]. IOT has great potential in Agriculture. Consider the future vision of the food lifecycles are well recorded from seeds, cultivation, products, transportation, food processing, sales in supermarket, it is exciting to have public confidence on food security and tremendous additional value to the agriculture and food suppliers.

However, in the agriculture internet of things, the data are sourced from different organizations and information technology facilities; it is an enormous challenge to integrate them into a workable system so that the midstream firms and the end consumers can query the history of the agricultural products unhindered by the bounds of the previous venders. One of the most important requirements of such integration is that the data semantics are consistence among the different phase of the products. To achieve such target, a unified ontology of agriculture products should be utilized by all the information systems of the different phases. Ontology is a new 
concept that is emerging from the various Semantic Web initiatives, which roughly speaking can be defined as a semantic system that contains terms, the definitions of those terms, and the specification of relationships among those terms.

In this paper, we proposed AgOnt - agriculture ontology for the purpose of agriculture internet of things. To keep the ontology light-weighted, we ignore the complexity of the specific agriculture activities or food processing; only the environments of the agriculture products are paid close attention to so that all the history can be queried by the follow-ups users. The remainder of the paper is organized as follows: section 2 presents the design of proposed AgOnt ontology. In section 3 the query of AgOnt based knowledge base is discussed. Section 4 highlights existing related work briefly. Section 5 concludes the current work and discusses possible future avenues for this research.

\section{AgOnt Ontology}

Ontology gives formal description on the hierarchical categories for real world knowledge [4], [5], [6]. Our approach uses ontology to capture the semantics of agriculture grains and their cultivation, logistics, storage history in the application of agriculture IOT. The purpose of building this ontology is to provide a mechanism for semantic interoperation between different systems (clouds) in the global agriculture clouds computing system. The semantic integration based on the ontology provides a solid basis for the integration of heterogeneous agriculture information system to form a huge information platform to record the grain lifecycles from the seeds, plant cultivation to food consumption.

AgOnt is based on the IEEE Suggested Upper Merged Ontology (SUMO) [7], which is the largest formal public ontology in existence today and used widely for research and applications in search, linguistics and reasoning. To capture the semantics of the grain or food lifecycle terminologies, we not only are able to define the environment of a product, but also describe the recall relationship between the succeeding forms such as the seeds and the plant, the wheat and the ponder, etc.

\subsection{Structure of the Ontology}

We create AgOnt ontology on the basis of two kinds of relationships in the lifecycle of agriculture products. One is the relationship of a product and its properties such as location, timestamp, environments parameters, processing status, etc. The other is the relationship between a product and its source products such as a plant and its seedlings where the plant grows from. Currently, we have defined 3 relationships between entities in our ontology generation: Is-a, Has-property, and Source-from:

- Is-a: entity $\mathrm{A}$ is an instance of entity B.

- Has-property: entity A has a property B.

- Source-from: entity A sources from entity B.

To describe and maintain the knowledge cleanly, we identify 5 main primitive domain classes as the top level ontology of the AgOnt as in Fig. 1. 
- Product class is used to describe the instance of agriculture products such as seeds, wheat, ponder, etc. It is the core of the whole ontology, which is a reflection of the view of thing-focused in the internet of things.

- Phase is a simple class to capture the conception of position in the lifecycle of the agriculture product. Phase is a property of any product.

- Time class is a supplement for phase to describe the concrete timestamp of the product activity. It is a property of any product.

- Location class is to capture where the product is and what organization is responsible for the maintenance of the agriculture phase

- Condition class captures the environment parameters of the product.

"Thing" is a dummy root for all classes in the ontology hierarchy. To illustrate the relationship "source-from", Fig. 1 gives an example between "Seed" and "Seedling" to show the grow-up or evolution of agriculture product. It is obvious that "Seed" and "Seedling" are the second level ontologies.

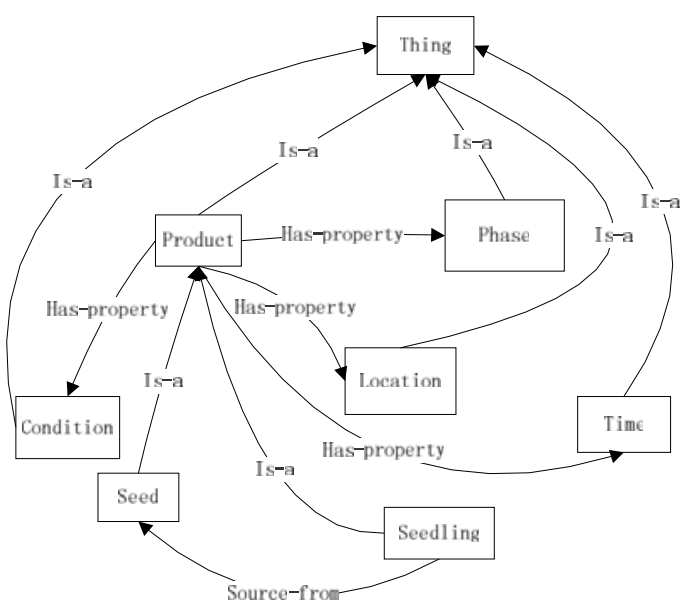

Fig. 1. The Top Level ontology of AgOnt

The Product class hierarchy is showed in Fig. 2. All agriculture products are dived into 5 subclasses, Seed, Seedling, Plant, Crop and Processed food, with each sources from previous. Crop capture the concept of the product cropped directly from the field. Different from it, Processed food describe the product produced by food processing factories.

The Phase class describe the abstract activity of the product ignore the specific characteristics. Currently we have 6 kinds of phases showed in Fig.3.

The Condition class captures all the sensor output to $\log$ the environment data of the product. Depending on the phase, the conditions of a product may have different properties. These property data are captured by smart sensors and can be propagated into the IOT. Fig. 4 shows a snippet of the conditions. 


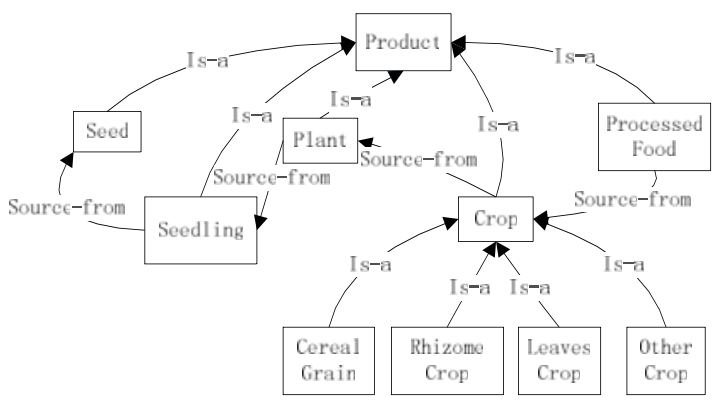

Fig. 2. The extended Product ontology

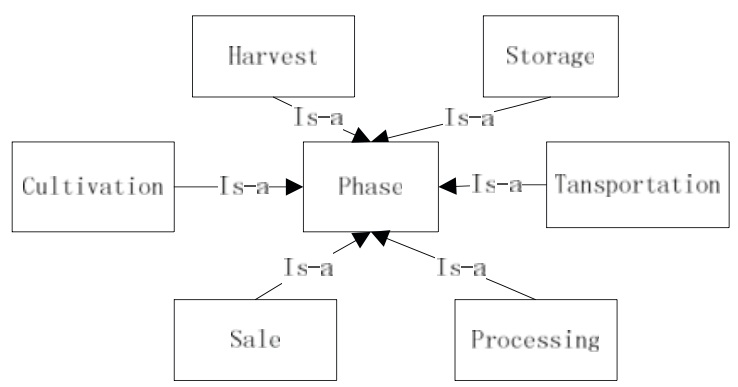

Fig. 3. The extended Phase ontology

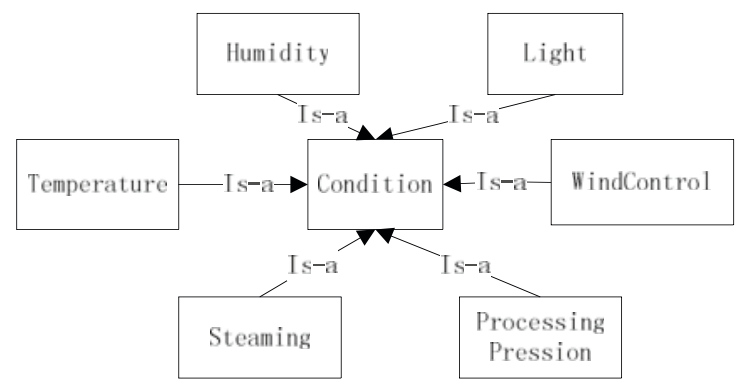

Fig. 4. The extended Condition ontology

\section{Querying on Agriculture Knowledge Base Based on AgOnt Ontology}

The AgOnt ontology provides a logical base where the semantics of the history of an agriculture product. Different agriculture information systems can integrate into a self-consistent knowledge base via a semantic middleware showed in Fig. 5. 


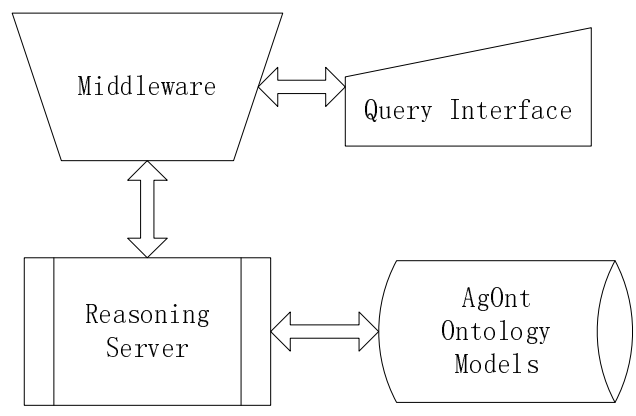

Fig. 5. Reasoning on the knowledge base

A user can query the abnormal condition history of a product after the knowledge base is built based on the ontology. The knowledge base has two kinds of descriptions. One is the classification of the agriculture terminologies and their relationship, the users and machines can reason and analyze the structure association of agriculture concepts. The other is the concrete instances of the products, their conditions and relationship, where the users and machines can judge what the history condition caused an unsatisfied product at the end. For example, assume at some phase a decayed product $\mathrm{ABP}$ is found, the user wish to identify where the problem comes from. Depending on the setting of abnormal condition, the user can query the history to find which phase may cause it. Assume the abnormal condition is "the temperature $>4 \operatorname{degC}$ and the humidity $>30 \%$ ", following query procedure is built to identify the problem.

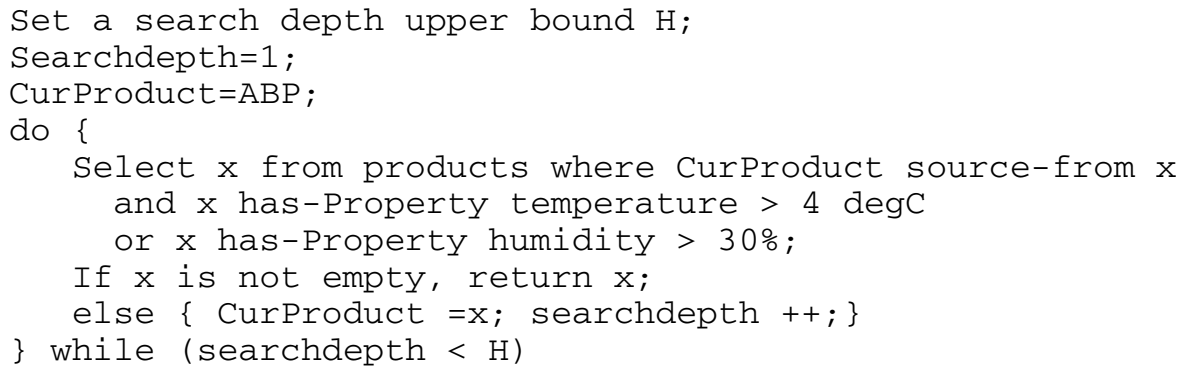

\section{Related Work}

In the agricultural sector there exist already many well-established controlled vocabularies, such as FAO's AGROVOC Thesaurus [8]. However, to build a semantic tool entirely effective on the Internet, there is a need to re-assess the traditional "thesaurus" approach and move towards to the development of "ontologies". Taking FAO's multilingual thesaurus AGROVOC as a starting point, the AOS Concept Server [9] is a project for such purpose with helping structuring and standardizing agricultural terminology to be used in a wide range of systems in the agricultural domain. The Concept Server will provide a core ontology in the domain of agriculture 
that people can take as a starting point for building more detailed domain specific ontologies. Although it is a really huge data store in agriculture, the server is more informatics-oriented than food lifecycles tracking.

Xie et al. [10] presented an agriculture-specific ontology to meet the requirement of agricultural knowledge processing and discussed the method for agricultural knowledge acquisition and representation.

Gangemi et al. [11] aimed at building an ontology in the fishery domain through the conceptual integration and merging of existing fishery terminologies, thesauri, reference tables, and topic trees. The ontology will support semantic interoperability among existing fishery information systems and will enhance information extraction and text marking, envisaging a fishery semantic web.

In summary, above ontologies are not helpful from the view of agriculture IOT application, where more semantic interoperability between grain or food lifecycles is focused. It is necessary to create a specific ontology suitable for the integration of multiple data sources of multiple phases so that all history record can be recalled.

\section{Conclusion and Future Work}

The AgOnt ontology gives a description of the concepts of agriculture terms and lifecycle between seeds, grains, transportations, storage and consumption. According to the classified domain terms and definition based on attribute descriptions in the ontology, a semantic middleware can reason on the food healthy knowledge. By using this proposed approach, the distributed agriculture products' information system can be accessed seamlessly. Thus follow-up users can know the history of the product before making a further processing. And the mechanism can also be used to recall when and where may take responsibility when a problem is found.

The future work is to find mechanisms of managing the agriculture ontology at runtime, to build a user query model to map various user requirements to reasoning procedures, and to sum up the experience of migration from the legacy systems to an ontology-based system.

\section{Acknowledgement}

Project supported by the National High Technology Research and Development Program of China (2008AA01Z208 and 2009AA01Z405), the National Natural Science Foundation of China (60772150), and the Youth Foundation of Sichuan Province (2009-28-419) and the Applied Basic Research Program of Sichuan Province (2010JY0013).

\section{References}

1. Katasonov, A., Kaykova, O., Khriyenko, O., Nikitin, S., Terziyan, V.: Smart Semantic Middleware for The Internet Of Things. In: 5th International Conference on Informatics in Control Automation and Robotics, pp. 169-178. INSTICC, Madeira, Portugal (2008) 
2. Yan, L., Zhang, Y., Yang, L.T.: The Internet of Things: from RFID to the Next-Generation Pervasive Networked Systems. Auerbach Publications, FL (2008)

3. Brock, D., Schuster, E.: On the Semantic Web of Things. In: Semantic Days 2006, Stavanger, Norway (2006)

4. Henson, C., Neuhaus, H., Sheth, A., Thirunarayan, K., Buyya, R.: An Ontological Representation of Time Series Observations on the Semantic Sensor Web. In: 1st International Workshop on the Semantic Sensor Web, Herkalion, Greece, pp. 79-94 (2009)

5. Kuhn, W.: A Functional Ontology of Observation and Measurement. In: Janowicz, K., Raubal, M., Levashkin, S. (eds.) GeoS 2009. LNCS, vol. 5892, pp. 26-43. Springer, Heidelberg (2009)

6. Fensel, D., Lausen, H., Polleres, A., de Bruijn, J., Stollberg, M., Roman, D., Domingue, J.: Enabling Semantic Web Services. Springer, Heidelberg (2007)

7. Niles, I., Pease, A.: Towards a Standard Upper Ontology. In: 2nd International Conference on Formal Ontology in Information Systems, pp. 2-9. ACM Press, New York (2001)

8. AGROVOC Thesaurus, http: //www. fao.org/agrovoc

9. AGROVOC Concept Server Workbench, http://naist.cpe.ku.ac.th/agrovoc

10. Xie, N.F., Wang, W.S., Yang, Y.: Ontology-based Agricultural Knowledge Acquisition and Application. In: 2nd IFIP International Conference Computer and Computing Technologies in Agriculture, vol. 1, pp. 349-357. Springer, Heidelberg (2008)

11. Gangemi, A., Fisseha, F., Pettman, I., Pisanelli, D.M., Taconet, M., Keizer, J.: A Formal Ontological Framework for Semantic Interoperability in the Fishery Domain. In: ECAI 2002 Workshop on Ontologies and Semantic Interoperability, pp. 16-30. IOS Press, Amsterdam (2002) 